\title{
Numerical modeling of fracture during nanoindentation of the TiN coatings obtained with the PLD process
}

\author{
K. PERZYŃSKI* and Ł. MADEJ \\ AGH University of Science and Technology, Department of Applied Computer Science and Modeling, \\ 30 Mickiewicza Ave., 30-059 Cracow, Poland
}

\begin{abstract}
In order to improve mechanical, frictional or biocompatibility behavior of well know materials like titanium (Ti) or titanium nitride (TiN) scientists are trying to develop new manufacturing and processing operations. One of those methods, that provide interesting results, is called deposition process. During deposition a material is upgraded with new surface layers that are characterized by specific required properties. These layers have usually different mechanical properties in comparison with a substrate material. A combination of different properties of the deposited layers can significantly change behavior of the structure under an exploitation condition. However, layers have usually nanometer scale, which causes problems with performing standard plastometric tests. One of the possibilities to solve this issue is an application of specially designed tests like nanoindentation. Nanoindentation can provide valuable information regarding mechanical and strength behavior of nanostructure components. These investigations are of importance to properly identify and design properties of the mentioned deposited materials. Unfortunately, experimental analyses at these scales are usually very expensive. That is why Authors decided to develop a numerical model of the nanoindentation test to investigate material behavior under loading conditions that can support experimental research.

The overall aim of this research is development of a failure model, which can take into account morphology of microstructure of ceramic TiN layer deposited on the silicon substrate. Modeling of crack behavior was realized on the basis of the extended finite element method (XFEM).
\end{abstract}

Key words: crack, nanoindentation, nanolayers, TiN, XFEM.

\section{Introduction}

Improving mechanical parameters of various materials requires a very specific treatment during manufacturing and processing stages. Various process temperatures, complex plastic deformation paths or different pressures during production are only some of the possibilities that can be applied in modern manufacturing facilities. In case of modern materials for bioengineering applications one of the best ways to obtain a product with a combination of required properties is to apply a deposition procedure. The main representative of this method is a Pulsed Laser Deposition (PLD) method. PLD is a modification of the standard Pulsed Vapour Deposition (PVD) approach. The main idea of the PLD is based on a high-power laser beam that is focused periodically onto the target material provoking instantaneous evaporation and ionization of the surface atoms. Products of such ionization consolidate atoms, electrons, and ions, which are driven away from the target plate at high speeds into the precisely controlled vacuum. Finally particles strike with the high speed into the surface of the substrate materials start to nucleate and grow. Thin films grow with the same chemical composition as the evaporated material. This process gives possibilities for fast and efficient production of layers on different kind of materials. PLD approach is widely used in biomedical industry to obtain multi layer biocompatible materials for medical elements e.g.: implants, artificial heart chamber, heart valves etc.
[1-7]. These kinds of materials are often subjected to nanoindentation tests in order to evaluate their in use properties and probability of their failure. Failure analysis is of importance when biomedical applications are considered.

Nanohardness test gives possibilities to receive information about maximal level of deformation, which can be safely applied to the deposited structure without the danger of failure. Additionally information regarding mechanical parameters like Young modulus and nanohardness can be obtained. These parameters can be then used for determination of other parameters like crack toughness and then maximal crack strain in material. However, experimental procedure is highly expensive because of specific equipment that has to be used.

That is why the main aim of this work is identification of appropriate crack initiation and propagation parameters for the TiN layer by using numerical simulation. That way amount of costly experimental analysis can be minimized. Comparison of the experimental and numerical tests and obtained crack parameters is presented within this article.

\section{Nanoindentation test}

2.1. Mechanical and strength parameters in nanoindentation. Basic properties of deposited layers contain set of information e.g. strength, ductility, crack resistance etc. All of those parameters can be calculated from data obtained during mentioned earlier nanoindentation test. Nanoindentation is

*e-mail: kperzyns@agh.edu.pl 
similar to conventional hardness test but performed in a much smaller scale. Maximal displacement of the indenter during this test reaches only nanometer range [8] (Fig. 1).

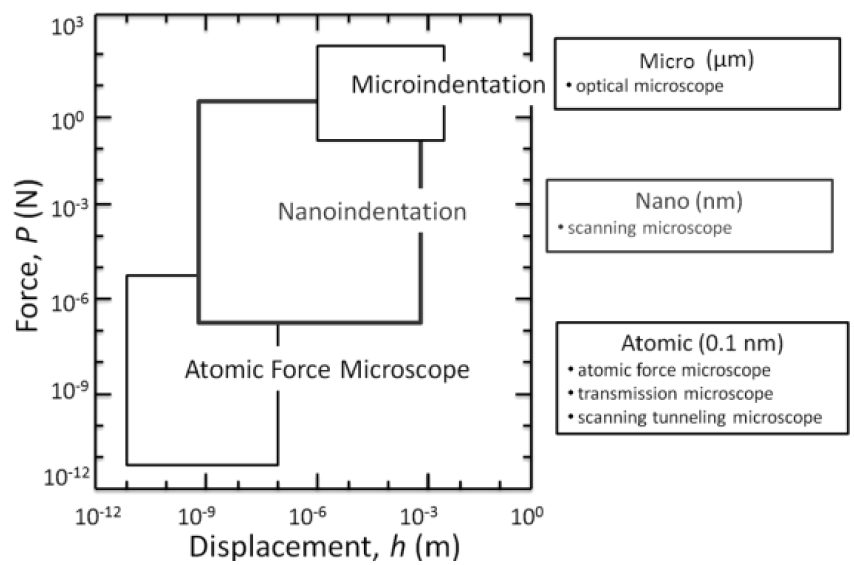

Fig. 1. Range of forces and displacements that can occur in indentation tests after Ref. 8

The indenter in the nanoindentation test can have different shapes, which can be selected from a vide range of possible solutions: Vickers, Berkovich or Conospherical. The commonly used Vickers indenter is applied in the present research (Fig. 2).

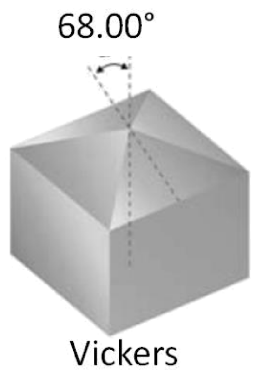

Fig. 2. Vickers indenter

Primary information about an investigated material in this test is described by the load-displacement curve. Information contained from such curve provides parameters related with single layer: strength, hardness, maximum crack load or number of pop-ins cracks. Usually the curve is obtained during loading and unloading stages of the indentation test. A schematic illustration showing formation of the loaddisplacement curve during nanoindentation process is presented in (Fig. 3).

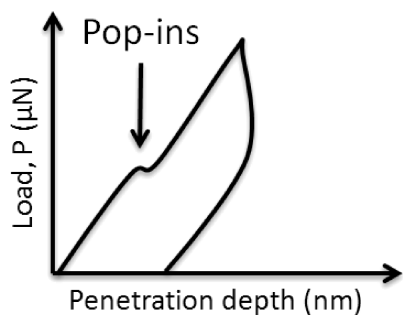

Fig. 3. Typical load-displacement curve obtained during nanoindentation test
Elastic properties of investigated structure are calculated by using combination of elastic properties of the indenter and elastic parameters of the specimen. Equation (1) describes the approach used to calculate elastic parameter in the nanoindentation test:

$$
\frac{1}{E_{r}}=\left(\frac{\left(1-\nu^{2}\right)}{E}\right)_{\text {specimen }}+\left(\frac{\left(1-\nu^{2}\right)}{E}\right)_{\text {indenter }},
$$

where $E_{r}$ is the combined elastic modulus of the indenter and material.

Nanohardness is a measure of how resistant the material is to various kinds of permanent shape changes when a force is applied. Nanohardness in nanoindentation is defined as the force applied to the contact area:

$$
H N=\frac{P_{c}}{A},
$$

where $P_{c}-$ maximum applied load during the nanoindentation, $A$ - the contact area between indenter and a sample. When Vickers indenter is used during the research the contact area can be described by:

$$
A=\frac{d^{2}}{1.8544}
$$

where $d$ is the maximum length of the area's secant after indentation with Vickers indenter.

During a nanoindentation test quite severe deformation is observed in the corners of the indenter working area. As a result these locations are under high risk of the crack initiation. In the literature these kinds of cracks are referred to as radial cracks [9]. This type of damage can be observed especially when investigated material is characterized by a brittle behavior. After nanoindentation test size of the existing radial cracks can be measured. This parameter combined with the Young modulus of the specimen, nanohardness and maximum indenter force gives a possibility to calculate crack toughness parameter (4):

$$
K_{c}=\alpha\left(\frac{E}{H N}\right)^{1 / 2}\left(\frac{P}{c^{3 / 2}}\right),
$$

where $\alpha$ is a constant for Vickers indenter equal to $0.016, E$ is Young modulus of the material, $H N$ is the nanohardness, $P$ is force applied by the indenter, $c=a+l$ is a length of radial crack. Schematic illustration of the area with radial cracks after Vickers nanoindentation is shown in Fig. 4.

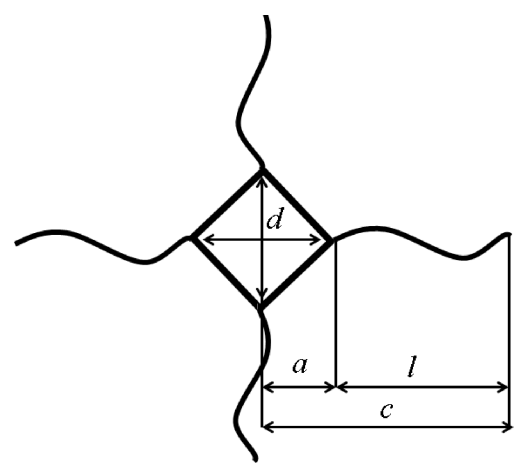

Fig. 4. Schematic illustration of Vickers nanoindenter working area and radials cracks initiated in the corners 
Crack toughness parameter is very helpful in describing mechanics of the crack in the material and provides also possibilities for verification of the numerical model with the experiments as presented in the next part.

2.2. Experimental results. During the development and identification stages of the numerical model of failure authors used results of experimental nanoindentation test described in literature [10]. Main idea of the experiment was to perform a series of nanoindentation tests on single deposited layer on the substrate material: a single TiN layer deposited on the silicon substrate. The sample was subjected to loading condition by the Vickers indenter. When a critical load $\left(P_{c}\right)$ was reached radial cracks started to initiate and evolve in the material. For the thin film TiN critical load was between $0.2 \sim 2 \mathrm{~N}$. Crack initiated in the locations where the high stress intensity was observed. These cracks propagated during indenter penetration in the same manner as schematically presented in Fig. 4. Major materials parameters of the TiN single layer were evaluated based on these experimental results (Table 1).

Table 1

Basic materials parameters of the TiN single layer

\begin{tabular}{cccc}
\hline \hline Layer & $\lambda[\mathrm{nm}]$ & $E_{r}[\mathrm{GPa}]$ & $H N[\mathrm{GPa}]$ \\
\hline TiN & 937 & 151.9 & 11.19 \\
\hline
\end{tabular}

According to Eq. (1) an exact Young modulus value for single TiN was obtained: $159.3 \mathrm{GPa}$. Combination of this parameter with material nanohardness in Eqs. (2) and (3) gave an opportunity for calculation of the maximum length of deformed area after nanoindentation test. This value for the single layers is equal $d_{\exp }=18.205 \mu \mathrm{m}$. Finally the crack toughness (4) was evaluated assuming maximum force used by indenter equal to $2 \mathrm{~N}$. For TiN layer crack toughness $K_{c}$ was calculated as $1.65 \mathrm{MPa} \sqrt{m}$.

Obtained experimental data were then used during the identification stage of parameters of the developed numerical model of material failure. The model and mentioned identification procedure are described below.

\section{Numerical model of nanoindentation test}

3.1. Model preparation and deformation results. A numerical model based on the experimental procedure of the nanoindentation test was developed in the commercial ABAQUS package. Nanoindentation is a process, where indenter has only local impact on the investigated sample. That is why Authors decided to use some simplifications and take into account only a small portion of the entire sample. As a result assembly of the investigated material consists of the two main parts. The first is the silicon substrate with the height of $19.063 \mu \mathrm{m}$. The second represents deposited TiN layer with the height equal to $0.937 \mu \mathrm{m}$. Vickers indenter tool is set above investigated sample with the sharp tip radius of $0.1 \mu \mathrm{m}$. All parts are assembled on the flat tool representing ground material. Indenter and plate are assumed to be the rigid bodies. Sample consisting of silicon with TiN layer is discretized with 220000 C3D 4-node linear tetrahedron elements. Model assembly with applied FE mesh is show in (Fig. 5).

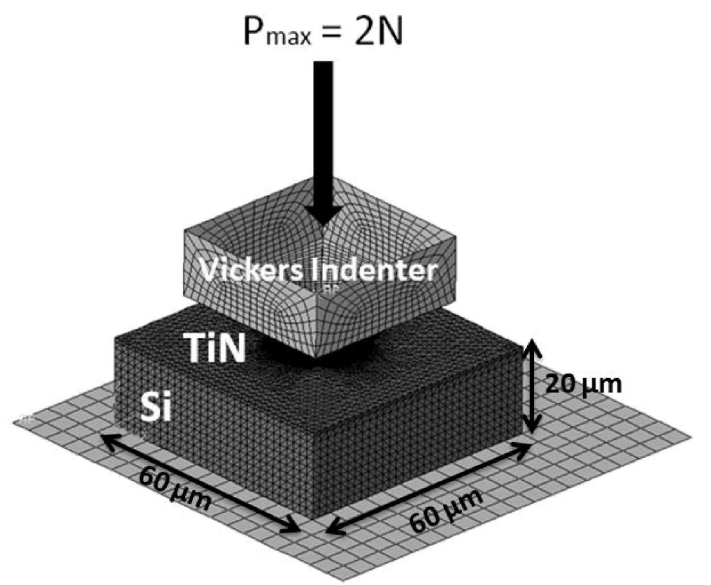

Fig. 5. Illustration of the assembly used in the numerical model

After definition of all parts in the assembly as well as generation of a mesh it is extremely important to choose appropriate material parameters. For the elastic material behavior a Young modulus $\left(E_{T i N}=151.9 \mathrm{GPa}, E_{S i}=144 \mathrm{GPa}\right)$ and the Poisson ratio $\left(\nu_{T i N}=0.3, \nu_{S i}=0.28\right)$ were selected for TiN and Si, respectively. During modeling step, the sample was hit by the indenter with load equal $\left(P_{\max }=2 N\right)$. In the end of the computational step maximal size of $d_{n u m}$ parameter reached the value of $17.766 \mu \mathrm{m}$. As seen in Fig. 6 developed numerical model provided good agreement with the experimental results.

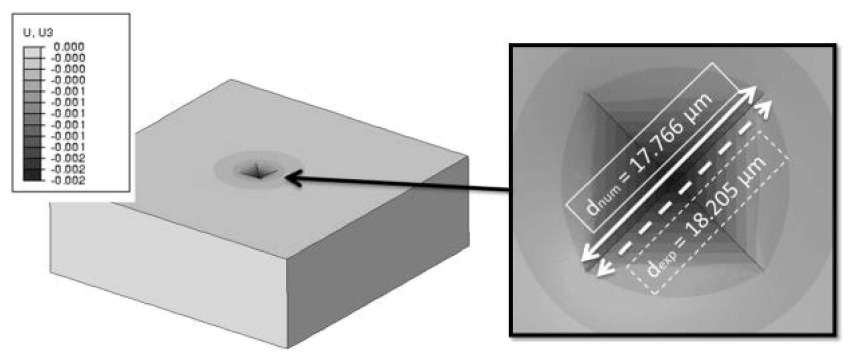

Fig. 6. Illustration of the indentation length after indentation process

As the model properly predicts real material behavior a numerical investigation of the crack initiation and propagation was realized using the extended finite element method.

3.2. XFEM method for modeling brittle cracks. For the investigation of the crack initiation and propagation during nanoindentation process numerical model presented in subsection above is modified with brittle crack model based on the XFEM (eXtended Finite Element) method. The XFEM is used for numerical modeling of processes where strong discontinuity can be found. This is the reason why the method is widely used for simulation of materials failure [11, 12].

The major assumption in the XFEM method is to consider the existence of a body $\Omega$ subjected to strong discontinuity conditions along the discontinuity path $S$. This discontinuity path is fixed at the reference configuration and characterized 
by the normal vector $\mathbf{n}$. The $S$ path is introduced in a way that it divides the $\Omega$ domain into $\Omega^{+}$and $\Omega^{-}$parts.

Additionally a Heaviside function $H_{S}(x)$ is defined on $\Omega$ :

$$
H_{S}(\mathrm{x})= \begin{cases}1 & \forall \mathrm{x} \in \Omega^{-} \\ 0 & \forall \mathrm{x} \in \Omega^{+}\end{cases}
$$

Discontinuity path $S$ where $\Gamma_{u}$ and $\Gamma_{\sigma}$ are the $\Omega$ boundaries subjected to the usual essential and natural boundary conditions, respectively, and they obey three conditions:

$$
\Gamma_{u} \cup \Gamma_{\sigma}=\partial \Omega, \quad \Gamma_{u} \cap \Gamma_{\sigma}=0, \quad \Gamma_{u} \cap \Omega^{h}=0
$$

$S_{h}^{+}$and $S_{h}^{-}$are the two boundaries of the $\Omega_{h}^{+}$and $\Omega_{h}^{-}$subdomains, which surround the $S$ path. It is also assumed that a function $\varphi^{h}(\mathrm{x})$ is defined as:

$$
\varphi^{h}(\mathrm{x})= \begin{cases}0 & \forall \mathrm{x} \in \Omega^{-} \backslash \Omega_{h}^{-} \\ 1 & \forall \mathrm{x} \in \Omega^{+} \backslash \Omega_{h}^{+}\end{cases}
$$

Additionally a unit jump function, which takes the zero value everywhere in $\Omega$ excluding $\Omega_{h}$, is introduced:

$$
M_{S}^{h}(\mathrm{x})=H_{S}(\mathrm{x})-\varphi^{h}(\mathrm{x})
$$

Such a function exhibits a jump across the discontinuity path $S$. An expression for the displacement field $u(x, t)$ under a strong discontinuity condition on $S$ is:

$$
u(\mathrm{x}, t)=\widehat{u}(\mathrm{x}, t)+M_{S}^{h}(\mathrm{x})[[u]](\mathrm{x}, t),
$$

where $\widehat{u}-$ the conventional part of the displacement field, $[[u]](x, t)$ - displacement jump function.

A finite element approximation of the strong discontinuity problem is illustrated in (Fig. 7), where $l_{e}-$ length of a straight line in the element, $n_{e}-$ the normal vector to $S_{e}$.

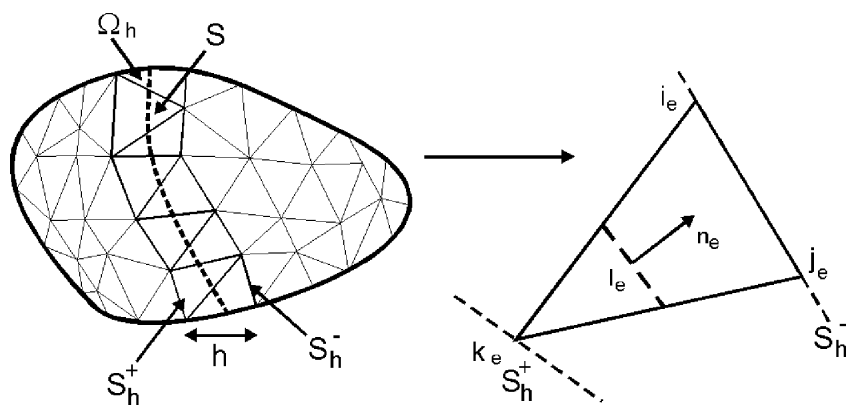

Fig. 7. Illustration of the finite element approximation of the discontinuity after Ref. 3

Finally the strain field is then calculated as it is shown in:

$$
\begin{gathered}
\varepsilon^{h}(\mathrm{x}, t)=\left(\nabla \widehat{u}^{h}\right)^{S}+\sum_{e=1}^{n_{e l}}\left(\nabla M_{S e}^{h} \times[[u]]^{h}(t)\right)^{S} \\
\Rightarrow \text { regular }+ \text { enhanced. }
\end{gathered}
$$

The more complex definition of using the XFEM method with nanolayers was described in other Authors work [3, 13].

One of the major drawbacks of the XFEM is a significant influence of mesh quality on final results. Different mesh sizes results in different crack propagation characteristics. This behavior is illustrated in (Fig. 8) by using simple FE model of tension. As seen application of the high mesh density in the XFEM method is of importance to obtain accurate results.

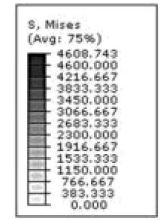

a)

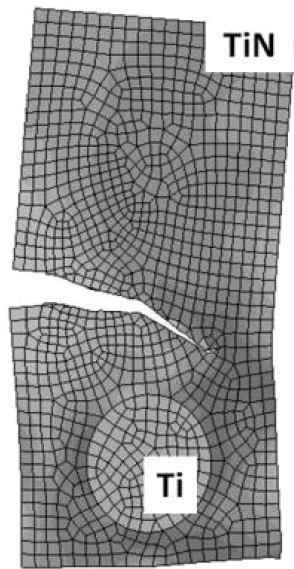

b)

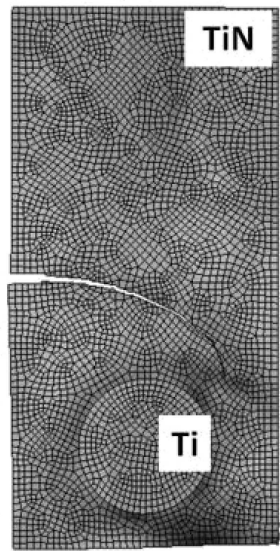

Fig. 8. Influence of the mesh density during simple tension test a) low mesh density b) high mesh density

3.3. Crack investigation during nanoindentation test. To model material failure Authors decided to use maximum principal strain criterion. Investigated material will start to crack when in some area during deformation the maximum strain reaches the critical value. Identification of this critical value is crucial in development of the reliable failure model. For this purpose an inverse analysis was used in the present research. From the real experiment it is possible to receive information regarding crack toughness $K_{c}$ and maximum crack length after indentation. The second value can also be obtained from the numerical model. That is why minimization of the discrepancies between experimental and numerical values of final crack length after indentation was used as a goal function during an inverse analysis. As a result a detailed critical value of strain that leads to crack initiation was evaluated. Schematic diagram of the applied inverse analysis procedure is presented in Fig. 9.

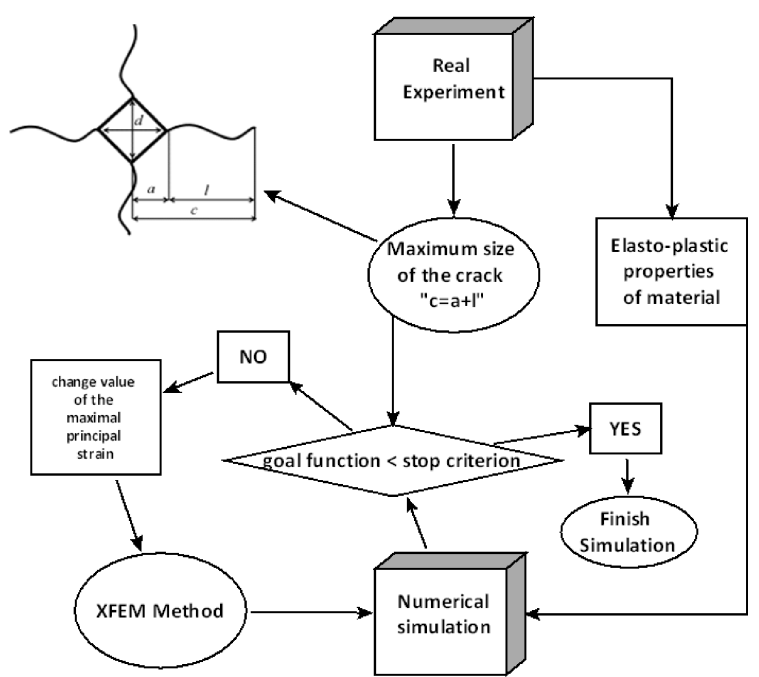

Fig. 9. Diagram representing inverse algorithm used in the present research 
Additionally to eliminate the effect of the mesh sensitivity the crack propagation is controlled by a displacement parameter. In this case crack will propagate when displacement of the crack tip is higher than minimal size of the smallest existing finite element in the model.

After a series of inverse algorithm steps a goal function reached assumed stop criterion. The calculated crack length for the TiN layer was equal to $\left(c_{\text {numerical }}=5.664 \mu \mathrm{m}\right)$ while experimental value was $\left(c_{\text {experimental }}=5.612 \mu \mathrm{m}\right)$. Such correspondence was obtained for maximum principal strain value equal to $\left(\varepsilon_{\text {crack }}=0.7\right)$.

As a result this value was used for the TiN layer as a crack initiation criterion in the developed numerical model. Examples of obtained results of the crack formation are shown in Fig. 10.

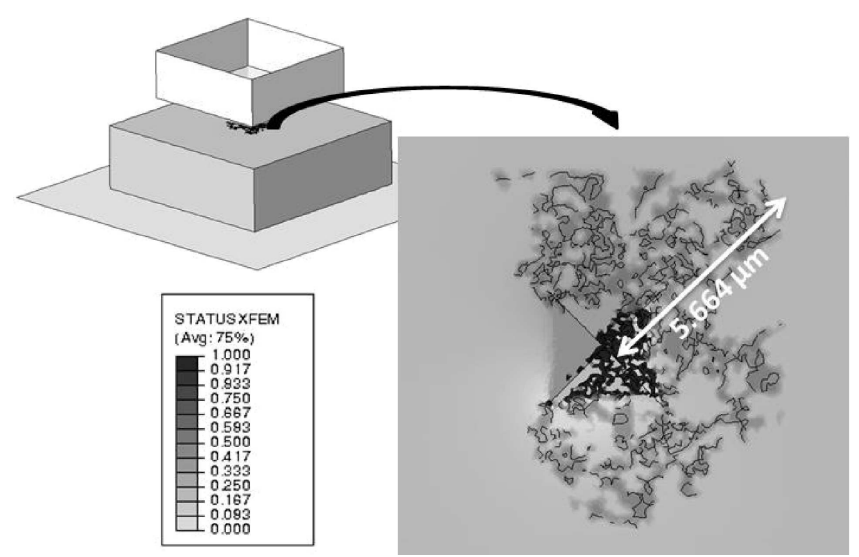

Fig. 10. Illustration of the size of the crack propagated after nanoindentation test

Based on the developed model it can be concluded that much more accurate data can be acquired from the simulation that from the experiments. Numerical approach gives possibility to observe not only processes occurring at the surface of the sample but also inside the specimen. Situation when crack propagates inside material body is extremely difficult to observe and measure experimentally. When numerical approach is used crack propagation in every time step can be easily analyzed as seen in Fig. 11.
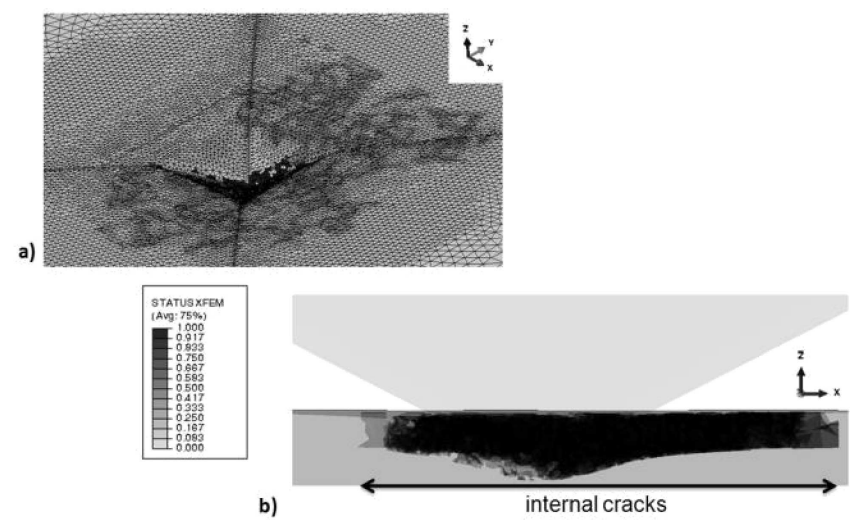

Fig. 11. Illustration of the a) surface cracks b) surface and internal cracks

\section{Conclusions}

Nanoindentation is a modern extension of the well-known method for testing the hardness of the material. This method can draw information from a much smaller area. Numerical modeling of nanoindentation gives new opportunities for design materials with deposited nanolayers. The possibility to receive similar mechanical properties in comparison with experimental results provides a new tool for the real process design. The ability for identification of zones where a crack can initiate gives the possibility to reduce the amount of experimental analysis. The possibility in modeling cracks in a digital material, in the nanoscale during the nanoindentation process has been presented in the paper. The developed numerical model:

- gives the opportunity to perform nanoindentation test in the virtual approach,

- is able to predict the crack path on the surface and inside the material,

- can be a direct model in the inverse analysis approach used during fracture model identification stages,

- can facilitate design of more complex combination of deposited structures, where crack propagation plays an important role.

Acknowledgements. The research is financed from the scientific grant number 15.11.110.151. FEM calculations were carried out in CYFRONET AGH by the grant number MNiSW/IBM_BC_HS21/AGH/076/2010.

\section{REFERENCES}

[1] C. Ratscha and J.A. Venables, "Nucleation theory and the early stages of thin film growth", J. Vacuum Science \& Technology A 21 (5), 96-109 (2003).

[2] R. Messier, A.P. Giri, and R.A. Roy, "Revised structure zone model for thin film physical structure", J. Vacuum Science \& Technology A 1, 500-503 (1983).

[3] K. Perzyński, Ł. Major, Ł. Madej, and M. Pietrzyk, "Analysis of the stress concentration in the nano-multilayer coating based on digital representation of the structure", Archives Metallurgy and Materials 56 (2), 393-399 (2011).

[4] K. Perzyński, Ł. Major, M. Kopernik, Ł. Madej, and M. Pietrzyk, "Analysis of the stress distribution in the nanogrid coatings based on digital representation of the structure", $M a$ terial Engineering 31 (3), 735-738 (2010).

[5] R. Major, P. Lacki, J.M. Lackner, and B. Major, "Modelling of nanoindentation to simulate thin layer behavior", Bull. Pol. Ac.: Tech. 54 (2), 189-198 (2006).

[6] M. Kopernik, A. Milenin, R. Major, and J.M Lackner, "Identification of materials model of TiN using numerical simulation of nanoindentation", Material Science and Technology 27 (2), 604-616 (2011).

[7] J.M. Lackner, L. Major, and M. Kot, "Microscale interpretation of tribological phenomena in Ti/TiN soft-hard multilayer coatings on soft austenite steel substrates", Bull. Pol. Ac.: Tech 59 (3), 343-355 (2011).

[8] M.L. Oyen, Handbook of Nanoindentation: with Biological Applications, Pan Stanford Publishing, Stanford, 2010. 
[9] B.R. Lawn, A.G. Evans, and D.B. Marshall, "Elastic/plastic indentation damage in ceramics: the median/radial crack system", J. American Ceramic Society 63 (9-10), 574-581 (1980).

[10] Z. Qi, "Processing, microstructure and mechanical behavior of nanocomposite multilayers", $P h D$ Thesis, University of Science and Technology, Beijing, 1999.

[11] T.T. Yu and Z.W. Gong, "Numerical simulation of temperature field in heterogeneous material with the XFEM", Archives Civil and Mechanical Engineering 13 (2), 199-208 (2013).

[12] T.T. Yu and P. Liu, "Improved implementation of the extended finite element method for stress analysis around cracks", Archives Civil and Mechanical Engineering 11 (3), 787-805 (2011).

[13] K. Perzyński and Ł. Madej, "Crack investigation of the multilayer TiN/Ti coatings during the nanoindentation test", Key Engineering Materials 504-506, 1293-1298 (2012). 